\title{
Pratiques
}

Linguistique, littérature, didactique

175-176 | 2017

Didactiques et médiations des arts et de la littérature

\section{Ce qu'ils voient, ce qu'ils disent quand ils rencontrent une œuvre}

What they see, what they say when they meet a work of art

\section{Christine Deronne}

\section{(2) OpenEdition}

\section{Journals}

Édition électronique

URL : http://journals.openedition.org/pratiques/3657

DOI : $10.4000 /$ pratiques.3657

ISSN : 2425-2042

Éditeur

Centre de recherche sur les médiations (CREM)

Référence électronique

Christine Deronne, «Ce qu'ils voient, ce qu'ils disent quand ils rencontrent une œuvre », Pratiques [En ligne], 175-176 | 2017, mis en ligne le 22 décembre 2017, consulté le 01 mai 2019. URL : http:// journals.openedition.org/pratiques/3657; DOI : 10.4000/pratiques.3657

Ce document a été généré automatiquement le 1 mai 2019.

(c) Tous droits réservés 


\title{
Ce qu'ils voient, ce qu'ils disent quand ils rencontrent une œuvre
}

What they see, what they say when they meet a work of art

\author{
Christine Deronne
}

1 Le développement des pratiques artistiques et culturelles est l'une des priorités gouvernementales inscrites dans la Loi d'orientation et de programmation pour la refondation de l'École de la République (juillet 2013). Cette volonté s'exprime dans un certain nombre de textes de cadrage: Le socle commun de connaissances, de compétences et de culture (BO $\mathrm{n}^{\circ}$ 17-23 avril 2015), Le parcours d'éducation artistique et culturelle (BO $n^{\circ} 28-9$ juillet 2015), les nouveaux programmes de l'école élémentaire applicables à la rentrée 2016 (BO spécial 26 novembre 2015). Par ailleurs depuis novembre 2005 Le Haut conseil de l'éducation artistique et culturelle, sous la présidence du ministère de la Culture et du ministère de l'Éducation nationale a pour mission d'assurer la promotion des arts à l'école. Le Projet de référentiel pour le parcours d'éducation artistique et culturelle - école primaire et college (décembre 2014) décline de façon précise les objectifs visés et propose des repères de progression pour l'ensemble des cycles de l'école primaire. Le document met en valeur trois piliers: le premier concerne les rencontres avec les cuvres, les artistes, les lieux de diffusion et de création; le second vise les pratiques artistiques et le dernier concerne l'acquisition des connaissances, qu'il s'agisse des repères historiques, esthétiques, techniques, ou de l'appropriation d'un lexique spécialisé simple permettant d'exprimer des émotions esthétiques, un jugement ou encore de décrire et analyser une œuvre, de développer l'esprit critique. La préoccupation des deux ministères concernant le développement culturel est également mise en évidence par une Charte pour l'éducation artistique et culturelle autour de 10 principes qui fondent l'éducation artistique et culturelle. Elle complète les grandes directives définies dans le Parcours d'éducation artistique et culturelle. L'éducation artistique et culturelle est à la fois éducation à l'art et par l'art, elle «permet aux jeunes de donner du sens à leurs expériences et de mieux appréhender le monde contemporain». La nouvelle organisation du temps scolaire depuis la rentrée 2014 et la mise en place d'activités culturelles et artistiques pour les enfants scolarisés contribuent également au développement culturel en lien avec les 
partenariats mis en place et les projets des acteurs locaux. Ce projet d'éducation artistique et culturel, tout à fait séduisant mais très ambitieux devrait se réaliser au moins une fois par cycle dans le cadre d'un projet élaboré par l'enseignant et réunissant les trois piliers précédemment évoqués. L'ensemble de ces directives officielles interroge les pratiques enseignantes. Les enseignants du premier degré (auxquels je m'intéresserai plus particulièrement dans le cadre de cet article) se trouvent confrontés à une série de questions didactiques qui ne sont pas neuves mais que les textes de préconisation rendent plus explicites.

2 Les objectifs du pilier 1 enjoignent aux enseignants de diversifier et multiplier les situations de rencontre avec les œuvres, les artistes, les lieux de diffusion de la création selon une progression sur les quatre cycles. Cet objectif de formation se traduit en une diversité de scénarios possibles répondant à des visées différentes : s'approprier les lieux, comprendre les codes qui les régissent, se comporter en spectateur, éduquer le regard, exprimer des émotions devant les œuvres regardées, etc. Parmi les grands objectifs de ce pilier, notre équipe de recherche a privilégié comme objet d'étude la réception de l'œuvre d'art. Si la rencontre peut avoir lieu en classe par l'intermédiaire des reproductions, ou de l'usage des sites web spécialisés, nous avons privilégié la rencontre dans les lieux dédiés spécifiquement à la diffusion de la création artistique, en musée ou galerie, c'est-à-dire la rencontre "face à face » avec l'œuvre dans toute sa réalité. La question de la réception n'est pas nouvelle. Elle a été particulièrement traitée par la didactique de la littérature (par exemple Dufays, Gemenne \& Ledur, 2005). L'analyse de situations scolaires et non scolaires de lecture a interrogé les tensions entre le sujet lecteur dans son rapport intime à l'œuvre et le sujet didactique dans son rapport scolaire à l'œuvre. La question des rapports qu'entretiennent les dimensions affectives et subjectives de la lecture avec les dimensions scolaires et formelles fondées sur des modélisations de théories de l'analyse littéraire a fait l'objet de nombreux débats. Les notions de lecture participative et lecture distanciée ont désigné deux composantes complémentaires de la lecture littéraire. Ces problématiques ont fait l'objet de nombreux travaux diffusés notamment par les Rencontres des chercheurs en didactique de la littérature. Le rapprochement, dans une perspective didactique, entre la littérature et les autres formes d'arts a donné lieu récemment aux $17^{\text {es }}$ rencontres des chercheurs en didactique de la littérature en juin 2016 à l'ENS de Lyon.

3 Notre recherche s'inscrit dans la continuité de travaux antérieurs (Deronne, 2011; 2012). Partant du constat que le temps réservé à la réception des œuvres (littéraires ou plastiques) est faiblement représenté en classe, nous visons à expliciter les significations que recouvre l'expérience de la rencontre avec une œuvre d'art (Parcours d'éducation artistique et culturelle, Pilier 1) et à montrer l'importance de ce moment dans le développement de l'éducation artistique. Dans le cadre d'un dispositif de recherche Lieux d'éducation associés (LÉA) défini dans le programme de l'Institut français de l'éducation (IFÉ), notre équipe ${ }^{1}$ a questionné la part de l'émotion et celle de l'analyse dans le moment de rencontre. Du point de vue méthodologique nous avons défini plusieurs protocoles de rencontre réalisés au Ccentre Pompidou-Metz (février 2015, exposition «Phares »²) et au Préau, espace d'art et de création de l'École supérieure du professorat et de l'éducation (ESPÉ) (février 2016, exposition « Nuits blanches» de S. Lécuyer). Plusieurs situations didactisées de rencontre ont été proposées en direction d'élèves de CP-CE1-CM1-CM2. Les matériaux recueillis sont nombreux : captations vidéo, enregistrements, carnets de bord, écrits des élèves, préparations et documentation des enseignants, recueil de données et 
expérimentation d'étudiants Master MEEF. Nous n'évoquerons que quelques fragments. Ils nous permettent d'analyser les pratiques langagières observables et les discours produits entre élèves ou entre adultes et élèves : que nous disent ces paroles d'élèves sur leur relation à l'œuvre d'art? Qu'en est-il de ce que les programmes désignent par l'émotion, la sensibilité, la perception, le jugement esthétique?

Dans un premier temps nous évoquerons les tensions et interrogations que soulève la question de la rencontre dans les espaces dédiés à l'exposition et à la réception : impact des lieux, légitimité et rôle des acteurs. Nous essaierons ensuite de préciser les notions qui ont alimenté notre réflexion : émotion, émotion esthétique, plaisir, jugement de gout. Nous terminerons par l'analyse de quelques situations didactiques qui visent à installer la relation de l'élève à l'œuvre et à favoriser l'émergence d'une parole sur l'œuvre, entre souci de susciter l'expression de la singularité de la réception et celui de donner accès aux connaissances artistiques et culturelles. Nous privilégierons l'analyse des interactions verbales entre élèves ou adulte-élèves.

\section{La rencontre au musée/en galerie : changer de lieux et de rôles}

\section{Les lieux : la classe/le musée}

5 La rencontre au musée implique d'amener les élèves dans un univers régi par des règles et des modes de fonctionnement différents de celui de la classe. Le volume de l'espace, la disposition des œuvres, la logique d'exposition prévue par le commissaire d'exposition imposent au visiteur des déplacements et des mouvements qui ne sont pas habituels pour des élèves de l'école élémentaire peu familiers de ces espaces. Le déplacement des élèves dans ces lieux répond à une ou plusieurs intentions pédagogiques différentes de la part des enseignants en direction des élèves : s'agit-il de fréquenter et comprendre les lieux, de s'accoutumer aux conduites de visiteurs, de découvrir un artiste, un courant ou une technique en lien avec des programmes d'histoire des arts? Clarifier les enjeux de la visite contribue à définir les dispositifs et les attendus. Et la visite des lieux s'impose avant toute scénarisation de visite pour les élèves.

6 La fréquentation d'une œuvre en classe est différente de celle qui a lieu en musée : les deux univers conditionnent des pratiques culturelles différentes. Le mode de consommation n'est pas le même. L'école, soucieuse de rentabilité, privilégie les temps d'analyse et d'interprétation de l'œuvre et l'acquisition de savoirs au regard des programmes, de ce fait elle scolarise la rencontre. L'œuvre d'art introduite en classe par le biais de reproduction (vidéoprojecteur ou photocopie) est davantage un objet scolaire de travail qu'une œuvre d'art à contempler. Le musée, au contraire, met en scène l'œuvre et lui confère son statut d'œuvre d'art (Goodman, 2006, p. 100) :

À vrai dire, un objet devient précisément une œuvre d'art parce que et pendant qu'il fonctionne d'une certaine façon comme symbole. Tant qu'elle est sur la route, la pierre n'est d'habitude pas une œuvre d'art, mais elle peut en devenir une quand elle est donnée à voir dans un musée d'art. Sur la route elle n'accomplit en général aucune fonction symbolique. Au musée, elle exemplifie certaines de ses propriétés par exemple, les propriétés de forme, couleur, texture.

7 C'est parce qu'elle est exposée au regard du spectateur que l'œuvre fonctionne comme œuvre, elle est activée par une mise en espace, un éclairage, des dispositifs 
d'encadrement et d'installation. La promenade du visiteur au musée n'est pas contrainte par le temps, le visiteur peut s'intéresser aux cartels ou les négliger, s'arrêter devant une œuvre ou l'ignorer, revenir sur ses pas.

\section{Les acteurs}

\section{L'enseignant/le médiateur}

8 Même s'il est davantage pris en compte dans la diversité de ses identités, comme le montre l'ensemble des travaux consacrés à la lecture littéraire évoquant par exemple l'importance du "sujet lecteur » dans la démarche interprétative des textes, l'élève, en classe, reste celui à qui on enseigne des savoirs. La lecture et l'analyse d'une œuvre nécessitent qu'il possède les connaissances techniques et esthétiques utiles pour produire un discours organisé sur l'œuvre, qui tend à ressembler à ceux que peut produire un spécialiste de l'histoire des arts. Les injonctions des programmes, les objectifs à atteindre, par exemple dans le domaine de l'histoire des arts, les volumes horaires consacrés à ces enseignements contraignent parfois les pratiques enseignantes et les orientent vers une démarche transmissive qui ne laisse que peu de place à l'émergence du discours de l'élève sur l'œuvre, à la fois singulier et partagé dans la classe. C'est l'enseignant qui apporte les savoirs et les ressources nécessaires à l'apprentissage des notions. La maitrise des savoirs disciplinaires artistiques est sécurisante pour l'enseignant, elle lui confère l'expertise. Dans une situation de découverte et de rencontre réalisée en classe, le maitre organise la gestion de la parole des élèves dans le but de la guider et de l'orienter vers l'objet d'apprentissage visé. Les gestes professionnels sont normés et anticipés. La parole de l'enseignant domine, les interactions sont réglées. Les enseignants expérimentés avec lesquels nous avons travaillé ont souvent témoigné de la sécurité que leur apporte en classe la phase de préparation et de conception d'une séance de rencontre avec une œuvre: recherches biographiques sur un auteur, catalogues, maitrise du lexique de spécialité concernant la technique et l'interprétation d'une œuvre, recherches sur les courants artistiques... Autant d'informations qui leur permettent de faire face à la diversité des éventuelles questions des curieux...

9 À l'inverse l'émergence de paroles spontanées, naïves face à l'œuvre dans un espace différent de celui de la classe inquiète et insécurise : il est difficile de s'emparer de cette parole qui renvoie à un agglomérat d'univers très différents les uns des autres : celui des expériences esthétiques, de la sensibilité, des connaissances sur le monde, des souvenirs, de l'imaginaire, des connaissances scolaires, des pratiques culturelles familiales.

Comment l'univers du ressenti sensible et de l'expérience esthétique de la rencontre s'expriment-ils ? Comment «la sensibilité », « l'ouverture aux émotions suscitées par les œuvres", "le partage des émotions ", "l'enrichissement de ses perceptions", "une émotion esthétique et un jugement critique » (extrait du Domaine $5 \mathrm{du}$ socle commun de connaissances, de compétences et de culture, $\mathrm{BO} \mathrm{n}^{\circ} 17-23$ avril 2015) peuvent-ils s'exprimer, s'apprendre et constituer des objets d'étude identifiables pour l'enseignant?

La dimension affective et subjective de la rencontre inquiète car elle signifie la singularité et l'impossibilité ou la difficulté à objectiver une rencontre au sein du groupe d'élèves. Parions qu'une des clés possibles de la compréhension de la dimension affective de la rencontre consiste pour l'enseignant à quitter son rôle d'enseignant pour endosser celui $\mathrm{du}$ visiteur/du spectateur et se laisser surprendre par les œuvres. Il pourra peut-être ainsi 
passer de la "posture de contrôle » dans l'organisation et la programmation des tâches auprès des élèves à une posture d'accompagnement « qui propose une aide en fonction de l'avancée des difficultés de la tâche ou encore une posture de "lâcher-prise" qui laisse aux élèves la responsabilité de leurs choix dans la façon de traiter les questions » (Bucheton \& Soulé, 2009).

Si l'enseignant a toute légitimité dans sa classe, qu'en est-il lorsqu'il se déplace au musée où le médiateur culturel est précisément un acteur essentiel dans le musée pour donner accès aux œuvres? Comme l'indique M.-C. Bordeaux (2008, p. 1), le développement des médiations culturelles est relativement récent:

la loi du 4 janvier 2004 relative aux Musées de France mentionne explicitement la médiation parmi les attributions des musées: "Chaque musée de France dispose d'un service ayant en charge les actions d'accueil des publics, de diffusion, d'animation et de médiation culturelles. Ces actions sont assurées par des personnels qualifiés ».

13 Le métier de médiateur culturel est défini dans le Répertoire national des certifications $(\mathrm{RNCP})^{3}$ :

[Sa] mission est d'organiser le rapprochement entre, d'une part, la culture et ses différentes formes de représentations et, d'autre part, les publics. C'est un spécialiste qui vise à favoriser leur rencontre en permettant au plus grand nombre l'accès à la culture, que ce soit d'un point de vue physique, social ou intellectuel. Il doit pouvoir recevoir et interpréter ce qui émane des uns et des autres, afin de concilier les deux parties et d'assurer une médiation en vue de sensibiliser le public, de l'initier, ou de le former.

14 Le médiateur culturel a donc pour mission de réduire la distance entre le public et l'œuvre. Lorsque l'enseignant décide d'emmener sa classe au musée, il se trouve face au choix d'accompagner lui-même le parcours de ses élèves et d'organiser une forme de médiation, mais il peut également la confier au médiateur.

Cependant médiateur et médiation ne se confondent pas et :

le terme «médiation » désigne, sur le terrain, des pratiques, des compétences et des fonctions particulières. Elles peuvent être classées selon trois axes : les médiations directes, de face-à-face, interactionnelles, entre professionnels de la culture et publics; les médiations indirectes, différées qui sont portées par des textes, des outils, des répertoires de démarches; les différentes techniques de gestion et d'administration de projet qui concourent à l'organisation de la rencontre. (Bordeaux, 2008, p. 8).

16 L'enseignant qui accompagne ses élèves dans l'objectif de faciliter la relation à l'œuvre d'art prévoit en amont des actions de médiations de la même façon que le ferait le médiateur. Il quitte, en partie seulement sans doute, son rôle d'enseignant pour devenir médiateur. Ce n'est donc pas l'identité professionnelle de celui qui installe la relation à l'œuvre qui importe dans la réussite d'une médiation (professeur-médiateur - voire l'artiste lui-même) mais l'intention qui préside à la médiation et les modalités mises en œuvre.

17 La médiation culturelle en musée recouvre donc une pluralité de pratiques en direction d'une grande diversité de publics visant à faciliter le passage d'une appréhension (saisie) première des œuvres d'art à une appréhension enrichie, développée dans le contexte d'une éducation artistique. Elle installe un rapport dialectique entre rencontre singulière et rencontre partagée (confrontations, réajustements), rencontre sensible et rencontre réfléchie. 


\section{L'élève/le spectateur} œuvres qui s'y trouvent. En fonction des pratiques culturelles familiales, les lieux lui sont plus ou moins familiers. Il découvre un espace sacralisé, isolé de l'extérieur, un enchainement de salles que l'on traverse selon un schéma préétabli et au sein duquel le corps se place avec plus ou moins d'aisance. Il passe d'un espace dans lequel sa place et son rôle d'élève sont définis en lien avec les apprentissages scolaires à un espace où il devient spectateur/regardeur : il exerce son regard et tisse des liens entre ce qu'il voit et ce qu'il est. Le spectateur émancipé (Rancière, 2008, p. 19) est un spectateur actif :

L'émancipation, elle, commence quand on remet en question l'opposition entre regarder et agir [...]. Elle commence quand on comprend que regarder est aussi une action [...] Le spectateur aussi agit, comme l'élève ou le savant. Il observe, il sélectionne, il compare, il interprète. Il lie ce qu'il voit à bien d'autres choses qu'il a vues, sur d'autres scènes, en d'autres sortes de lieux. Il compose son propre poème avec les éléments du poème en face de lui.

L'activité de l'élève dans le musée est cependant rarement libérée de toute contrainte. Les responsables des musées souhaitent que les élèves soient encadrés pour éviter tout dommage. Les règles de sécurité exigent que des accompagnateurs encadrent de petits groupes d'élèves. Les conditions de la visite déterminent donc les modes de conduite. De ce fait l'enseignant met en place un dispositif d'accompagnement qui laisse une plus ou moins grande autonomie à l'élève. Lors de notre visite de l'exposition "Phares », nous avons pu remarquer cette diversité d'attitudes: certains élèves étaient entièrement accaparés par la découverte des lieux et l'immensité de l'espace, d'autres n'ont pas hésité à se déplacer, s'asseoir pour prendre des notes.

\section{Pluralité des rencontres}

Nous faisons l'hypothèse que, quel que soit son bagage culturel, l'élève face à une œuvre peut produire un discours à partir de ce qu'il voit de l'œuvre et que pour faciliter l'émergence de cette parole "naïve ", il n'est pas nécessaire de s'en remettre "à l'avis autorisé de "spécialistes en blouse blanche" dans une abdication du jugement individuel et du libre arbitre » (J.-H. Martin [2012] cité par P. Baryga [2015]). L'expression première de la perception sensorielle d'une œuvre d'art passe par la vue et le mouvement corporel qui accompagne le regard vers l'œuvre (avancer, reculer, regarder de biais). Cette première appréhension qui passe par les filtres de la perception du spectateur s'accompagne de réactions spontanées qui sont des manifestations d'émotions ordinaires : joie, étonnement, intérêt, plaisir, inquiétude. L'objet regardé provoque ces émotions qui peuvent s'exprimer et se partager au sein d'un petit groupe de spectateurs.

21 Mais quel est le rapport de cette première expression spontanée d'émotions ressenties face à l'œuvre avec l'expérience esthétique, l'émotion esthétique et la dimension cognitive du rapport à l'œuvre? Ces notions font débat et donnent lieu à des positions très opposées.

Nous ne ferons pas, dans le cadre de cet article, l'état de l'art de la question au centre des préoccupations des philosophes et des sociologues, nous aborderons quelques points de vue nous permettant de mesurer l'importance de la question, d'éclaircir un certain nombre de tensions et de situer les démarches dont il sera question.

Pratiques, 175-176 | 2017 


\section{Émotion ordinaire/émotion esthétique}

\section{conceptions conflictuelles de l'expérience esthétique et le rapport entre l'affectif et le} cognitif. Majoritairement, pour l'auteure, les théoriciens privilégient le cognitif.

On aurait pu penser que l'intérêt porté par bon nombre de théoriciens contemporains à la question de l'expérience esthétique, qu'elle soit nommée ainsi ou "attitude» chez Monroe Beardsley, "activité " chez Nelson Goodman, "attention" chez Gérard Genette, ou encore «conduite» chez Jean-Marie Schaeffer, confère une nouvelle actualité à la question de l'émotion esthétique. Or ce n'est pas le cas. Le choix des mots qu'utilisent ces auteurs montre éloquemment que l'intérêt porté à l'attitude mentale qui caractérise l'expérience esthétique s'accompagne d'un désintérêt pour la dimension affective de cette même expérience. L'élément affectif de celle-ci est négligé, omis, voire nié. (ibid., p. 2)

L'étude de C. Talon-Hugon s'appuyant sur le genre poétique et la poéticité distingue émotion ordinaire et émotion esthétique. L'émotion ordinaire comme la joie ou la colère est une émotion qui «surprend, déconcerte, assiège et paralyse momentanément l'esprit » (ibid., p. 3), elle concerne le sujet tout entier, en traduisant la façon dont il est touché par l'objet, elle implique l'individu dans sa vie ordinaire et son rapport au monde. L'émotion esthétique détache momentanément le sujet de sa condition ordinaire. L'œuvre provoque des émotions autres que celles suscitées par le monde ordinaire et qui ne se confondent pas avec l'état du sujet: «l'émotion poétique nous ravit un instant hors de notre condition » (ibid., p. 9).

Dans un article récent C. Talon-Hugon (2016) développe la notion d'émotion esthétique dans une perspective historique en se penchant sur la peinture. Elle constate que la notion d'émotion esthétique a évolué étant donné le passage de ce qu'elle nomme du pathétique artistique classique et romantique à l'émotion esthétique moderniste. L'émotion pathétique est provoquée par la représentation des situations et des personnages figurant sur les tableaux, elle donne l'occasion de ressentir « de manière extraordinaire des passions ordinaires » (ibid., p. 3). On rapprochera de ce type d'émotion celle que les élèves ont exprimée lors de la visite de l'exposition «Phares» face aux œuvres de Y. Pei Ming (Les survivants).

L'émotion esthétique, quant à elle, est provoquée par des "qualités sensibles", les formes, les couleurs, les matériaux.

À la différence des émotions ordinaires, cette émotion proprement esthétique n'est pas suscitée par des événements pathétiques ou des êtres passionnés, mais par des couleurs et des formes. Ces couleurs et ces formes ne sont pas ici des moyens de représenter des contenus pathétiques : elles sont, en elles-mêmes, objets d'émotion. ( ibid., p. 4)

L'auteur conclut cependant que cette fracture entre deux conceptions de l'émotion (ordinaire-pathétique et esthétique) ne peut valoir pour toutes les productions artistiques :

Il est sans doute légitime de ne chercher qu'un plaisir spécifiquement esthétique dans Azur de Mallarmé ou dans une composition abstraite de Kandinsky. Il est beaucoup plus discutable de vouloir interdire les émotions ordinaires au lecteur du Dormeur du val de Rimbaud ou au spectateur de La Mort de Socrate de David. (ibid., p. 9) 

psychologie pour préciser ce qu'est l'émotion. Le projet de l'auteur n'est pas de comprendre «l'expérience des œuvres d'art dans sa (leur) spécificité, mais l'expérience esthétique dans son caractère générique» (ibid., p.12). Pour J.-M. Schaeffer toute expérience esthétique se traduit par l'attention au monde qui nous met en relation avec les objets, suscite l'émotion et procure le plaisir. L'émotion esthétique «fait partie des modalités de base de l'expérience commune du monde [...] elle exploite le répertoire commun de nos ressources attentionnelles, émotives, hédoniques, mais en leur donnant une inflexion non seulement particulière, mais bien singulière » (ibid.). Plus loin il précise que l'expérience esthétique est caractérisée par « l'ancrage dans nos ressources cogntives et émotives de base et l'usage qui en est fait » (ibid., p. 18). Par ailleurs, l'auteur insiste sur la continuité cognition/émotion :

Il ressort que la cognition et les émotions forment deux systèmes structurellement corrélés. Les situations dans lesquelles l'un des systèmes est activé sans que l'autre le soit sont extrêmement rares [...] il n'existe en revanche pas de processus émotionnel dépourvu de dimension cognitive : comme on vient de le voir, toute émotion est le produit direct ou indirect [...] d'une évaluation cognitive, même si elle n'est pas toujours consciente ni toujours fiable (ibid., p. 141).

La corrélation émotion/cognition dont il est ici question a souvent été au cœur des rencontres de nos élèves avec l'œuvre. L'immensité des œuvres présentées et leur étrangeté ont provoqué beaucoup d'étonnement, d'admiration mais aussi de questionnement sur la réalisation, la technique, les significations.

\section{Plaisir esthétique/plaisir artistique}

Pour d'autres comme N. Everaert-Desmedt (2010) qui s'appuie sur la théorie de l'activité de réception de Ch. S. Peirce, la réception artistique est étroitement liée à un processus cognitif qui conduit au plaisir artistique. Plaisir artistique et plaisir esthétique ne se confondent pas : le plaisir esthétique peut être provoqué par une série d'objets du monde qui nous entoure alors que le plaisir artistique vise à découvrir le « beau en soi » :

La réception artistique n'est pas une perception spontanée d'une qualité de l'œuvre, ni une contemplation, mais une cognition, un processus cognitif. C'est dans l'activité cognitive elle-même que réside le plaisir artistique : plaisir de sentir qu'il y a quelque chose à comprendre, qu'on est sur le point de comprendre, que quelque chose d'absolument nouveau est sur le point de devenir intelligible, que l'on va découvrir du possible. (ibid., p. 4).

Le plaisir artistique résiderait donc dans l'activité cognitive de l'interprétation. L'œuvre d'art a une signification " qui vaut la peine d'être cherchée et que le récepteur s'attend à découvrir ». L'interprétation de l'œuvre résulte d'une alternance entre observations et hypothèses interprétatives fondées sur les connaissances du sujet. Elle dépend du degré d'attention du spectateur et des connaissances culturelles, techniques dont il dispose mais également des expériences sensorielles sollicitées plus particulièrement dans certaines formes artistiques comme par exemple dans les installations artistiques contemporaines. Les élèves de CP face à l'œuvre d'A. Kapoor (Sans titre) ont manifesté leur étonnement et leur plaisir : ils n'ont pas hésité à se déplacer, à bouger et à s'amuser des effets que produisaient leurs mouvements sur la demi-sphère concave rouge. Ils ont 
cherché, ont formulé des hypothèses, rapproché l'œuvre d'objets de leur quotidien, et n'ont pas manqué de questionner leurs enseignants.

\section{Réception non informée/réception informée}

\section{l'œuvre et à une approche de la compétence artistique. Les observations sur les publics de} spectateurs aboutissent à la distinction entre deux modes de réception :

Dès lors, la compétence artistique fournit une échelle sur laquelle se positionnent les différents modes de réception. La réception naïve, non informée, tend par exemple à ne voir dans les œuvres que les objets représentés, et juge les matériaux de représentation à partir de critères extra-artistiques (qualité de la facture, etc.). À l'inverse, la réception informée de ce code adéquat reconnait l'iconographie de l'œuvre, et s'efforce de replacer sa création dans son contexte, en la situant dans l'histoire de l'art, de l'opposition des genres, ou encore dans la biographie du peintre lui-même. (ibid., p. 77)

Mais « il faut admettre que certains actes sémiques qui constituent la réception, pour le public, n'ont pas ce caractère d'interprétation » (ibid., p. 75). Et S. Coavoux évoque par exemple les signes d'étonnement, les interjections face aux œuvres :

L'observation des visiteurs de musées d'art montre au contraire un rapport souvent distant : arrêts brefs, marques d'approbation ou de désapprobation sèches (" c'est beau!", «c'est affreux!») qui relèvent du «geste » plus que du discours, selon l'admirable mot de Wittgenstein, incapacité fréquente à parler du tableau autrement qu'en décrivant ce qu'il représente. (ibid., p. 78)

Ces distinctions de niveau de réception interrogent le sociologue dans le contexte d'une réflexion plus large sur la démocratisation culturelle. Cependant l'opposition entre les deux modes de réception n'est sans doute pas aussi tranchée et ne se ramène pas à une opposition entre deux catégories sociales car l'individu est soumis à plusieurs influences qui font évoluer son comportement culturel.

Pour terminer cette présentation qui ne se veut pas exhaustive mais qui vise à préciser ce qui peut définir la relation de l'élève à l'œuvre d'art, l'émotion et le plaisir esthétiques, il nous faut explorer la notion de jugement de gout.

\section{Jugement de gout/jugement esthétique}

E. Aujaleu (1997) distingue appréciation esthétique et jugement esthétique. L'une renvoie au degré de satisfaction que procure la relation du spectateur à l'objet, à la satisfaction que procure la présence du beau ou le désarroi face au laid, à l'effroyable. Il s'agit donc d'un état. L'autre renvoie à un jugement de valeur objectivé. Se référant à I. Kant l'auteur exprime l'idée que le jugement de gout n'est pas un jugement de connaissance. Le jugement de gout est désintéressé, il s'appuie sur une expérience subjective donc singulière il renvoie au plaisir des sens, il est partagé par plusieurs. Donner la définition des critères du beau n'est cependant guère envisageable mais cette absence de scientificité n'exclut pas qu'au sein de groupes d'individus donnés un accord sur les critères d'évaluation du jugement de gout et de la sensibilité puisse s'instaurer par les relations intersubjectives.

Dans un autre domaine, celui de la didactique, J.-L. Dumortier (2006, p. 193) articule la question du jugement de gout à la didactique du français et à la réflexion sur les

Pratiques, $175-176 \mid 2017$ 
compétences visées dans les programmes de lecture écriture en Belgique. Il propose de distinguer « énoncer un jugement de gout motivé » et « énoncer un jugement de valeur argumenté ».

Énoncer un jugement de goût à propos d'une œuvre d'art, c'est faire état du (dé)plaisir éprouvé au contact de cette œuvre. Dire les motifs du jugement de goût, c'est dire pourquoi elle nous a plu ou déplu :

Énoncer un jugement de valeur à propos d'une œuvre d'art, c'est faire état d'une évaluation qui peut aussi bien procéder ou ne procéder pas du (dé)plaisir éprouvé à son contact. Argumenter un jugement de valeur, c'est tenter d'obtenir un accord, premièrement, sur des critères de valeur et, deuxièmement, sur la présence, dans l'œuvre considérée, de traits correspondant à ces critères. Cela indépendamment $\mathrm{du}$ fait que l'argumentateur avalise ou n'avalise pas ces derniers. En d'autres termes, argumenter un jugement de valeur, ce n'est pas forcément essayer de faire admettre à autrui que ce qui a ou n'a pas de la valeur pour nous-mêmes (parce que cela nous a plu ou déplu) devrait avoir la même valeur - voire quelque valeur - pour tout le monde ou, plus particulièrement, pour les personnes que nous nous efforçons de persuader. (ibid., p. 193)

\section{Des œuvres/des réceptions}

40 Certaines œuvres installeraient-elles plus facilement une communication en direction du spectateur, favoriseraient-elles davantage l'expression des sentiments et du jugement?

41 On serait tenté de dire qu'une œuvre figurative représentant des éléments du monde réel « touchera » plus facilement le spectateur car une relation d'empathie entre spectateur et objets ou situations représentées s'installera. Cependant tout dépend de l'objet représenté et de la connivence culturelle possible entre ce qui est représenté et le spectateur. Il n'est pas évident que certaines représentations bibliques ou historiques soient d'un accès immédiat pour les élèves car le contexte peut leur manquer. D'autre part, ce n'est pas parce qu'une œuvre est contemporaine et abstraite qu'elle suscitera moins d'émotions qu'une œuvre classique représentant de façon figurative une situation ordinaire. Lors de la visite de l'exposition "Phares", les tableaux de Y.Pei-Ming, Survivants, ont particulièrement provoqué l'émotion des élèves à la fois par la représentation de visages exprimant la souffrance et par le volume des œuvres, les nuances des couleurs sombres et des techniques du peintre.

\section{Quelques éléments de conclusion}

Le parcours précédent met en évidence des points de vigilance pour tout enseignant visant à développer l'appréhension et la réception des œuvres d'art en musée. Le passage de l'école au musée donne de nouveaux rôles aux élèves, mais ils ne sont sans doute pas entièrement différents. Dans le cadre de la visite scolaire au musée, l'enseignant accompagnateur emporte de l'école des pratiques professionnelles, les élèves au musée ne sont pas réellement des visiteurs autonomes mais gardent des comportements scolaires. Les outils de médiation proposés par le musée ou l'enseignant lors des visites sont assez proches des ressources pédagogiques de la classe. Enfin les notions relatives à la réception et fréquentation des œuvres méritent éclaircissement de façon à ce que les intentions des enseignants soient clarifiées pour chaque dispositif mis en place: qu'en est-il de l'émotion suscitée par les œuvres, de la continuité cognition/émotion? De l'interprétation symbolique? Du plaisir esthétique? De l'appréciation et du jugement 
esthétique? D'une réception naïve ou d'une réception informée? Que nous disent les paroles des élèves sur leur relation à l'œuvre d'art?

Dans les pages qui suivent nous analyserons deux dispositifs mis en œuvre lors de la visite de l'exposition «Phares » en 2015 et un dispositif réalisé lors de l'exposition «Épreuve » au Préau des Arts à l'ÉSPÉ de Lorraine. Chacun des dispositifs vise à installer la relation de l'élève à l'œuvre et à faire émerger la parole de l'élève sur l'œuvre dans la situation de rencontre en face-à-face.

\section{Ce qu'ils voient, ce qu'ils disent}

\section{Les dispositifs de la recherche}

En février 2015, environ 70 élèves CP-CE1-CM1-CM2 se sont rendus avec leurs enseignants et des accompagnateurs (parents, étudiants emplois d'avenir, professeur/stagiaire EFS) au Centre Pompidou-Metz.

Dix œuvres ont été sélectionnées par l'équipe pour faire l'objet du regard des élèves : Joan Miro, Personnages et oiseaux dans la nuit (1974); J. Le Parc, Déplacement du spectateur (1965/2013) ; Y. Pei-Ming, Survivants (2000) ; P. Soulages, peinture (1979) ; L. Nevelson, Réflexions of a waterfall I (1982); S. Hantaï, Tabula (1974); R. Delaunay, Entrée du hall des réseaux du palais des Chemins de fer (1937); F. Léger, Composition aux deux perroquets (1935-1939) ; A. Kapoor, Sans titre (2008) ; F. Stella, Polombe (1994).

Les élèves ont été répartis en 8 groupes. Chacun des groupes a reçu une consigne visant à faciliter l'attention des élèves devant les toiles. Trois groupes de CM2 disposent d'un carnet de route, ils n'ont pas bénéficié de préparation en classe avant la visite. Sur chaque carnet figurent la représentation couleur de l'œuvre, et sa légende. Le G1 doit prendre le temps de bien observer l'œuvre et d'indiquer dans son carnet ce qu'il en pense à partir d'enclencheurs comme "J'ai aimé cette œuvre parce que...", "Je n'ai pas aimé cette œuvre parce que... », "Cette œuvre est intéressante parce que... », « Cette œuvre me fait penser à... ». Ils gardent une trace de leur passage.

Le G2 dispose également d'un carnet de route. Sous chaque reproduction d'œuvre figurent deux ou trois questions, par exemple, à propos de l'Entrée du hall des réseaux $d u$ palais des Chemins de fer de R. Delaunay : «Ces panneaux ont été réalisés pour l'exposition internationale des arts et techniques en 1937. Ils étaient placés à l'entrée de deux pavillons (bâtiments) dédiés aux transports modernes. À ton avis, à quel type de transport Robert Delaunay veut-il faire penser? Quels sont les éléments des différents panneaux qui permettent de le dire ? Reproduis ces éléments sur la page de droite et une invitation à reproduire un fragment de l'œuvre ».

Le G3 dispose également d'un carnet sur lequel sont reproduites les questions des cartels à destination spécifique des élèves. Par exemple à propos du tableau Polombe de F. Stella :

Parcours de long en large la gigantesque toile de Franck Stella... Elle est tellement grande et tellement riche que ton regard ne peut englober toute l'œuvre. Tu dois au contraire te concentrer tour à tour sur de nouveaux détails.

Cette œuvre semble faite en trois dimensions.

Quelle technique l'artiste a-t-il employée pour parvenir à cette illusion?

A- la superposition de différentes formes très complexes crées par l'ordinateur

B- le collage de véritables spirales sur la toile. 
49 antérieure à la visite. Un autre groupe qui a bénéficié de préparation, assure la médiation en direction de $\mathrm{CP}$, un dernier groupe CM1-CM2 visite en autonomie avec un fichier reproduisant chaque œuvre et la consigne de noter de façon libre ce qu'il souhaite. il dispose d'un fichier avec des questions simples et des icones désignant les activités, par exemple « donner un titre au tableau, écris 2 ou 3 mots sur ce que tu ressens ».

51 L'ensemble des 8 groupes est invité à s'exprimer quelques semaines plus tard sur ce qui l'a marqué, sur ce dont il se souvient.

En février 2016 une nouvelle population de CM1-CM2 (2 classes) a pu se rendre au Préau pour visiter une exposition consacrée à différentes techniques de gravure. Des étudiantes fonctionnaires stagiaires du master Métiers de l'enseignement, de l'éducation et de la formation (MEEF) ont pu assurer une médiation.

La visite au Centre Pompidou-Metz a donné lieu à l'expression d'une série d'émotions liées à la découverte du lieu, du volume des salles, à la taille des œuvres. Les regards, les attitudes corporelles, les mimiques, les interjections traduisaient étonnement, admiration, émerveillement. Les œuvres de J. Le Parc et A. Kapoor ont suscité beaucoup de commentaires admiratifs. Les émotions esthétiques ont été également exprimées quant aux formes, couleurs, techniques, matériaux. Certains tableaux, notamment Survivants ont suscité une émotion pathétique provoquée par l'expression des visages. L'interprétation cognitive en fonction des connaissances culturelles et artistiques des élèves a également été enclenchée : des hypothèses interprétatives ont été élaborées (cf. à propos de Polombe) en faisant appel au vécu, aux références acquises, aux connaissances sur le monde. Enfin si le jugement de gout a été exprimé, la formulation d'un jugement de valeur argumenté a manqué dans bien des interactions observées ou dans les traces écrites. Face à des œuvres contemporaines peu familières de ce que les élèves rencontrent en classe, les élèves ne disposaient pas suffisamment de données pour étayer leur propos et aller au-delà d'une première appréhension naïve de l'oeuvre.

\section{Rencontres avec Polombe de F. Stella}

Les 3 études de cas qui suivent concernent les élèves de cycle 3 :

- Les premiers échanges des élèves de CM2 autour de l'œuvre F. Stella, Polombe, ainsi que les traces écrites correspondant.

- La visite assurée par la médiatrice sur la même œuvre auprès d'un groupe mixte CM1-CM2.

- Une visite réalisée au Préau des Arts par une étudiante fonctionnaire stagiaire pour des CM2.

Un groupe de CM2 devant Polombe de F. Stella

Ils disposent du carnet de route avec enclencheurs simples. 
Tableau 1. Diversité des liens tissés avec l'œuvre d'art

\begin{tabular}{|c|c|c|c|}
\hline intervenant & $\begin{array}{l}\text { Dialogue entre les } \\
\text { élèves }\end{array}$ & $\begin{array}{l}\text { Observations sur les } \\
\text { comportements des } \\
\text { élèves }\end{array}$ & $\begin{array}{l}\text { Analyse des liens tissés par les } \\
\text { élèves avec l'œuvre d'art }\end{array}$ \\
\hline E1 & Alors? & & \\
\hline E2 & $\begin{array}{l}\text { Ça fait penser à la vie, } \\
\text { les couleurs de la vie, } \\
\text { tu vois. Le blanc ya } \\
\text { rien de dépressif } \\
\text { dedans. Ya de tout }\end{array}$ & $\begin{array}{l}\text { E2 s'adresse à E1, } \\
\text { cherche son accord. }\end{array}$ & $\begin{array}{l}\text {-Description centrée sur les } \\
\text { couleurs, } \\
\text { Référence aux connaissances } \\
\text { personnelles, } \\
\text { Raisonnement par analogie } \\
\text { Dimension interprétative } \\
\text { (symbolique)- jugement de } \\
\text { gout. }\end{array}$ \\
\hline E1 & Ouais, ouais & $\begin{array}{l}\text { Il regarde, semble } \\
\text { approuver }\end{array}$ & \\
\hline E2 & $\begin{array}{l}\text { Là ya un peu de noir } \\
\text { aussi mais ça rend... }\end{array}$ & & Continuité de la description \\
\hline E1 & $\begin{array}{l}\text { Là ya plein de fil de } \\
\text { fer ça fait bizarre... }\end{array}$ & & $\begin{array}{l}\text { Évocation de la matière, } \\
\text { Constat-appréciation } \\
\text { esthétique-jugement de gout }\end{array}$ \\
\hline E2 & $\begin{array}{l}\text { Moi ça me fait penser } \\
\text { à la vie là, tout ce } \\
\text { qu'il peut y avoir. } \\
\text { Et c'est aussi grand. } \\
\text { La vie est grand. } \\
\text { C'est long... C'est pas } \\
\text { si long que ça. }\end{array}$ & $\begin{array}{l}\text { Affirmation de l'idée } \\
\text { Essai de } \\
\text { développement }\end{array}$ & $\begin{array}{l}\text { Répétition pour convaincre } \\
\text { mais pas de réelle } \\
\text { argumentation-commentaire } \\
\text { technique sur la quantité } \\
\text { d'objets représentés et la taille } \\
\text { du tableau-association avec la } \\
\text { vision symbolique de la vie (?) }\end{array}$ \\
\hline E1 & Ben quand même & $\begin{array}{l}\text { Reprise sur l'idée de } \\
\text { longueur }\end{array}$ & Réajustement -étonnement \\
\hline E2 & $\begin{array}{l}\text { Mais c'est quand } \\
\text { même aussi très long } \\
\text { de faire ça tout seul }\end{array}$ & $\begin{array}{llr}\text { Glissement } & \text { de } \\
\text { signification : } & & \\
\text { «long » } & & \text { se } \\
\text { rapportant } & & \text { à } \\
\text { l'espace et à } & \text { la } \\
\text { temporalité } & & \end{array}$ & $\begin{array}{l}\text { Evaluation du travail de } \\
\text { l'artiste-appréciation } \\
\text { artistique- }\end{array}$ \\
\hline E1 & $\begin{array}{l}\text { Ben tu vois la } \\
\text { superposition des } \\
\text { trucs }\end{array}$ & $\begin{array}{l}\text { Tentative d'apporter } \\
\text { une nouvelle idée }\end{array}$ & Technique de l'artiste \\
\hline
\end{tabular}




\begin{tabular}{|c|c|c|c|}
\hline E2 & $\begin{array}{l}\text { Moi je pense que ça } \\
\text { fait rapport avec la } \\
\text { vie }\end{array}$ & $\begin{array}{l}\text { Reprise avec force } \\
\text { de la première idée }\end{array}$ & $\begin{array}{l}\text { Du côté de l'interprétation- } \\
\text { valeur symbolique }\end{array}$ \\
\hline $\begin{array}{l}\text { Adulte } \\
\text { accompagnateur }\end{array}$ & $\begin{array}{l}\text { Ça te fait penser à } \\
\text { quoi }\end{array}$ & $\begin{array}{l}\text { S'adresse à un } \mathrm{E} \\
\text { assis- }\end{array}$ & \\
\hline E3 & Du collage & & Technique de l'artiste \\
\hline adulte & D'accord & & \\
\hline E2 & $\begin{array}{l}\text { Il y a de l'action. Ça va } \\
\text { un peu partout }\end{array}$ & $\begin{array}{l}\text { Ajout de l'idée de } \\
\text { mouvement }\end{array}$ & \\
\hline E1 & $\begin{array}{l}\text { Ya un gamin de } 3 \\
\text { mois }\end{array}$ & & \\
\hline E3 & $\begin{array}{l}\text { Voilà une personne } \\
\text { de } 3 \text { mois qui prend } \\
\text { des trucs et colle }\end{array}$ & & $\begin{array}{l}\text { Jugement esthétique- } \\
\text { dévalorisation }\end{array}$ \\
\hline E4 & Euh, trop de couleurs & $\begin{array}{l}\text { L'E s'approche de E1 } \\
\text { E2 }\end{array}$ & Appréciation esthétique \\
\hline E4 & $\begin{array}{l}\text { Et puis on dirait des } \\
\text { filets, des filets qui } \\
\text { attrapent, euh }\end{array}$ & & Hypothèse- (conditionnel) \\
\hline $\mathrm{E} 1$ & Je sais pas.. & & \\
\hline E4 & $\begin{array}{l}\text { Quelque chose, enfin } \\
\text { on sait pas quoi mais }\end{array}$ & & interrogation \\
\hline E1 & Un amas de, de ... & & $\begin{array}{l}\text { Tentative de rapprochement } \\
\text { de référents réels }\end{array}$ \\
\hline E4 & Quelque chose & & \\
\hline E1 & Un amas d'ordures & & \\
\hline Adulte & Tu écris tout ça? & & \\
\hline E1 & Un amas & & \\
\hline E1 & Finalement je sais pas & & renoncement \\
\hline E5 & $\begin{array}{l}\text { Non, mais en fait, } \\
\text { c'est vraiment des } \\
\text { papiers collés sur le } \\
\text { tableau! }\end{array}$ & $\begin{array}{l}\text { S'approche très } \\
\text { près-relance- }\end{array}$ & $\begin{array}{l}\text { Affirmation concernant la } \\
\text { technique de l'artiste }\end{array}$ \\
\hline
\end{tabular}




\begin{tabular}{|c|c|c|c|}
\hline adulte & $\begin{array}{l}\text { Pas si près! Touchez } \\
\text { pas! }\end{array}$ & $\begin{array}{l}\text { E5 pointe un stylo } \\
\text { pour prouver son } \\
\text { propos }\end{array}$ & \\
\hline E1 & $\begin{array}{l}\text { Ouais ça fait vraiment } \\
\text { des trucs superposés }\end{array}$ & & $\begin{array}{l}\text { Revient à son idée de départ } \\
\text { confirmée par l'observation de } \\
\text { E5. }\end{array}$ \\
\hline E2 & $\begin{array}{l}\text { Là ça fait penser à des } \\
\text { arbres. Là-bas ça fait } \\
\text { penser à un astéroïde. } \\
\text { Là ça fait penser } \\
\text { astéroïde, arbre, } \\
\text { plante végétation }\end{array}$ & En pointant du doigt & $\begin{array}{l}\text { Poursuite de l'idée de vie, } \\
\text { métaphore }\end{array}$ \\
\hline E1 & $\begin{array}{l}\text { On dirait qu'il est } \\
\text { près }\end{array}$ & & \\
\hline E2 & Tu vois l'eau, la mer & & \\
\hline E1 & $\begin{array}{l}\text { De loin tu vois les } \\
\text { effets et de } \\
\text { quand c'est fait. C'est } \\
\text { judicieux, r JU-DI- } \\
\text { CIEUSEMENT } \\
\text { fait! }\end{array}$ & & $\begin{array}{l}\text { Appréciation esthétique } \\
\text { tentative d'explication (« de } \\
\text { près-de loin ») }\end{array}$ \\
\hline E2 & $\begin{array}{l}\text { En fait des fois tout ce } \\
\text { que j'ai dit c'est j'l'ai } \\
\text { trouvé dans ma tête } \\
\text { et puis là-dessus. }\end{array}$ & $\begin{array}{l}\text { E montre le tableau } \\
\text { (là-dessus) }\end{array}$ & $\begin{array}{l}\text { Dimension méta sur la façon } \\
\text { de regarder }\end{array}$ \\
\hline
\end{tabular}

Deux élèves lancent chacun une piste : d'un côté la métaphore filée de la vie et de l'autre la technique utilisée : accumulation et superposition.

Par rapport aux échanges verbaux, les traces écrites sont très brèves $\mathrm{E} 5 \mathrm{:}$ « j’aime bien car c'est coloré et on dirait des papiers collés »; E2 : « ça fait penser à la vie, tout ce qu'il peut y avoir »; E4 : «je n'aime pas trop cette œuvre car elle a trop de couleurs. On dirait des filets qui attrapent des animaux »; E1 : « un amas de tout »; E3 : (pas de document écrit).

\section{La médiatrice du musée devant Polombe de F. Stella}

Les élèves se rassemblent autour de la médiatrice, s'assoient en demi-cercle, face au tableau de F. Stella. La médiatrice tourne le dos à l'œuvre. Les premières remarques portent sur les couleurs flashies du tableau.

Tableau 2. Médiation ou transmission?

\begin{tabular}{|l|l|l|l|}
\hline intervenant & Dialogue élèves - médiatrice & Observations & Analyse \\
\hline
\end{tabular}




\begin{tabular}{|c|c|c|c|}
\hline $\begin{array}{l}\text { M } \\
\text { (médiatrice) }\end{array}$ & $\begin{array}{l}\text { Est-ce qu'on a l'habitude de voir du fluo } \\
\text { dans les tableaux? }\end{array}$ & Question fermée & \\
\hline Es & Non, non & & \\
\hline E1 & C'est un tableau un peu unique & $\begin{array}{l}\text { Enchainement à } \\
\text { partir de la } \\
\text { réponse } \\
\text { précédente }\end{array}$ & \\
\hline M & C'est très rare & $\begin{array}{l}\text { Renforce } \\
\text { l'opinion- mais } \\
\text { n'explore pas } \\
\text { l'idée exprimée } \\
\text { par « unique » }\end{array}$ & \\
\hline E2 & $\begin{array}{l}\text { C'est un tableau un peu abstrait aussi } \\
\text { comme... }\end{array}$ & & \\
\hline $\mathrm{M}$ & $\begin{array}{l}\text { Ah très bien, bravo, c'est un tableau } \\
\text { abstrait }\end{array}$ & $\begin{array}{l}\text { Coupe la parole à } \\
\text { E2 }\end{array}$ & $\begin{array}{l}\text { Satisfaction de M } \\
\text { car il s'agit d'une } \\
\text { réponse attendue } \\
\text { qui guide son } \\
\text { propos }\end{array}$ \\
\hline E3 & $\begin{array}{l}\text { C'est un tableau aussi, comme on dit, on } \\
\text { dirait qu'on a découpé des morceaux de } \\
\text { feuilles qu'on les a collées }\end{array}$ & & $\begin{array}{l}\text { Hypothèse } \\
\text { concernant la } \\
\text { technique }\end{array}$ \\
\hline E2 & oh & & \\
\hline$M$ & $\begin{array}{l}\text { Et bien c'est un petit peu ce que disait } \\
\text { Hortense. Oui c'est ça }\end{array}$ & & $\begin{array}{l}\text { Pas de reprise sur } \\
\text { la technique au } \\
\text { moment où surgit } \\
\text { la question }\end{array}$ \\
\hline E1 .... & On dirait que & & Difficulté à dire \\
\hline M & $\begin{array}{l}\text { Tu sais plus.... Alors tout ce que vous } \\
\text { avez dit, c'est bien. En fait Franck Stella } \\
\text { est un artiste. Alors lui il est américain, } \\
\text { d'accord. Et il est toujours en vie. Il vit } \\
\text { toujours Franck Stella. Il travaille } \\
\text { toujours Franck Stella. Lui, il fait de très } \\
\text { grands tableaux, de toutes les couleurs, } \\
\text { avec plein de couleurs fluo et vous avez } \\
\text { raison quand il peint ses tableaux et } \\
\text { bien, c'est des feuilles qu'il imprime. Il } \\
\text { travaille même avec un imprimeur. C'est } \\
\text { quelqu'un qui est spécialisé pour } \\
\text { imprimer des choses. }\end{array}$ & $\begin{array}{l}\text { Transmission } \\
\text { d'informations et } \\
\text { de connaissances } \\
\text { sur l'auteur }\end{array}$ & Effet de saturation \\
\hline
\end{tabular}




\begin{tabular}{|c|c|c|c|}
\hline E3 & Il imprime ou il dessine? & & $\begin{array}{ll}\text { Suite } & \text { de } \\
\text { l'hypothèse } & \\
\text { concernant } & \text { la } \\
\text { technique } & \end{array}$ \\
\hline $\mathrm{M}$ & $\begin{array}{l}\text { IL découpe et après il les colle pour faire } \\
\text { des grands tableaux }\end{array}$ & $\begin{array}{l}\text { Continue } \\
\text { l'explication, sans } \\
\text { répondre }\end{array}$ & \\
\hline E4 & Et après il dessine la même chose? & & $\begin{array}{lr}\text { Nouvelle } & \\
\text { interrogation } & \\
\text { technique- } & \text { les } \\
\text { réponses } & \text { ne } \\
\text { donnent } & \text { pas } \\
\text { satisfaction- } & \\
\text { curiosité } & \text { pour } \\
\text { l'objet produit } & \end{array}$ \\
\hline $\mathrm{M}$ & $\begin{array}{l}\text { Chut chut E2 a dit que c'était abstrait. } \\
\text { Alors c'est vrai. Ça veut dire qu'on sait } \\
\text { pas ce que c'est. }\end{array}$ & $\begin{array}{lr}\begin{array}{l}\text { Décrochage } \\
\text { élèves, }\end{array} & \text { des } \\
\text { Rupture: } & \text { M. } \\
\text { abandonne } & \text { les } \\
\text { explications } & \\
\text { concernant } & \text { la } \\
\text { technique et } & \text { la } \\
\text { curiosité } & \text { des } \\
\text { élèves } & \end{array}$ & $\begin{array}{ll}\text { Apport } & \text { de } \\
\text { connaissances } & \end{array}$ \\
\hline & {$[\ldots]$} & & \\
\hline $\mathrm{M}$ & $\begin{array}{l}\text { C'est abstrait parce que F .S. il voulait } \\
\text { qu'on puisse imaginer quelque chose. Il } \\
\text { a voulu créer en quelque sorte des } \\
\text { mondes imaginaires. D'accord? C'est } \\
\text { pour ça que c'est abstrait, pour qu'on } \\
\text { puisse imaginer. D'ailleurs il a appelé } \\
\text { son tableau, regardez sur vos feuilles, } \\
\text { c'est quoi le titre de l'œuvre? Polombe }\end{array}$ & $\begin{array}{lr}\text { Apport } & \text { de } \\
\text { connaissances } & - \\
\text { définition } & \text { de } \\
\text { « abstrait » } & \end{array}$ & $\begin{array}{l}\text { Discours déclaratif } \\
\text { sur les intentions } \\
\text { de l'auteur }\end{array}$ \\
\hline Es & Polombe & & \\
\hline $\mathrm{M}$ & $\begin{array}{l}\text { En fait Polombe c'est le nom d'une ville } \\
\text { imaginaire dans un livre qui s'appelle } \\
\text { les voyages de Sir John Mandeville. Est- } \\
\text { ce que vous connaissez un livre } \\
\text { d'histoires qui parle d'un monde } \\
\text { imaginaire? }\end{array}$ & $\begin{array}{ll}\text { Passage de la } & \text { de } \\
\text { notion } & \\
\text { peinture } & \text { abstraite } \\
\text { à imaginaire- }\end{array}$ & $\begin{array}{l}\text { Apport } \\
\text { d'informations } \\
\text { culturelles qui } \\
\text { échappent aux } \\
\text { élèves } \\
\text { convocation des } \\
\text { référents culturels } \\
\text { des élèves }\end{array}$ \\
\hline E3 & Alice au pays des Merveilles & & \\
\hline
\end{tabular}


Le discours de la médiatrice au musée remplit une fonction précise, celui d'apporter des connaissances sur l'auteur et l'œuvre pour réduire la distance du spectateur à l'œuvre mais il provoque également un effet de saturation qui met les élèves à distance de ce qu'ils voient et cherchent à dire. Paradoxalement la médiation joue très peu son rôle.

\section{Rencontre avec les Nuits blanches de S. Lécuyer}

Extrait de la médiation réalisée par une étudiante fonctionnaire stagiaire (EFS) au Préau des arts (ESPÉ de Lorraine) sur l'œuvre de S. Lécuyer Nuits blanches. L'étudiante n'a pas fait de préparation spécifique concernant les techniques liées à la gravure ni au travail de l'auteure. Elle a pris du temps pour adopter la conduite d'un visiteur de musée, laisser venir l'œuvre à elle et s'interroger sur la part de l'affectif et du cognitif qui oriente son regard.

Tableau 3. Description et mise en intrigue

\begin{tabular}{|c|c|c|c|}
\hline & Dialogue EFS-élèves & Observations-remarques & $\begin{array}{l}\text { Analyse des liens tissés par } \\
\text { les élèves avec l'œuvre d'art }\end{array}$ \\
\hline EFS & $\begin{array}{l}\text { Alors je vais vous demander de } \\
\text { regarder les tableaux et de me } \\
\text { dire un petit peu ce que vous } \\
\text { voyez. Je vous laisse réfléchir un } \\
\text { petit peu, on prend le temps de } \\
\text { regarder. }\end{array}$ & $\begin{array}{l}\text { Les élèves sont très prés } \\
\text { de l'installation des } \\
\text { gravures- Incitation au } \\
\text { regard et à la description }\end{array}$ & \\
\hline E1 & $\begin{array}{l}\text { On voit une dame qui dort dans } \\
\text { un lit. }\end{array}$ & & description \\
\hline EFS & $\begin{array}{l}\text { Où ça? Où est-ce que tu vois } \\
\text { une dame qui dort dans un lit? }\end{array}$ & L'E1 s'approche & \\
\hline E1 & $\begin{array}{l}\text { Elle, et elle est déguisée en } \\
\text { fantôme. }\end{array}$ & & Première interprétation \\
\hline E2 & $\begin{array}{l}\text { Ouais elle c'est ce que j'allais } \\
\text { dire et là on dirait qu'elle a un } \\
\text { truc de mariée sur la tête. }\end{array}$ & & $\begin{array}{l}\text { Seconde hypothèse et } \\
\text { interprétation de ce qui est } \\
\mathrm{vu}\end{array}$ \\
\hline E3 & $\begin{array}{l}\text { Ouais ça c'est plus un truc de } \\
\text { mariée. }\end{array}$ & & $\begin{array}{l}\text { Adhésion à l'une des } \\
\text { hypothèses-tentative } \\
\text { d'argumentation } \\
\text { plus " }\end{array}$ \\
\hline EFS & $\begin{array}{l}\text { On dirait qu'elle a un truc de } \\
\text { mariée sur la tête. Alors } \\
\text { pourquoi tu dis qu'elle a un truc } \\
\text { de mariée sur la tête? }\end{array}$ & & \\
\hline
\end{tabular}




\begin{tabular}{|l|l|l|l|}
\hline E3 & $\begin{array}{l}\text { Parce que au Moyen Âge ils } \\
\text { avaient ça. (..) Avec des petits } \\
\text { fils comme ça pour cacher les } \\
\text { larmes }\end{array}$ & $\begin{array}{l}\text { Association avec des } \\
\text { représentations de tableaux, } \\
\text { des référents, tentative pour } \\
\text { situer le contexte de l'œuvre }\end{array}$ \\
\hline EFS & $\begin{array}{l}\text { Pour cacher les larmes ?? } \\
\text { c'est une dame du Moyen-âge ? } \\
\text { E1 la aussi y a une barque avec } \\
\text { de l'eau }\end{array}$ & $\begin{array}{l}\text { La description des éléments } \\
\text { qui figurent dans le récit } \\
\text { continue }\end{array}$ \\
\hline E2 & Avec un monsieur dessus & Personnage de l'intrigue \\
\hline E4 & $\begin{array}{l}\text { Là y a une dame dans la forêt } \\
\text { avec un loup je crois. }\end{array}$ & \\
\hline
\end{tabular}

\section{Un peu plus tard les échanges portent sur la couleur noire utilisée par l'auteure.}

\section{Tableau 4. Approche technique}

\begin{tabular}{|l|l|l|}
\hline EFS & $\begin{array}{l}\text { Qu'est ce qui a été utilisé là, comment ça a été } \\
\text { fait ça? }\end{array}$ & \\
\hline E & Avec une gravure & \\
\hline EFS & Avec une gravure? & $\begin{array}{l}\text { Tentative d'explication concernant les } \\
\text { techniques }\end{array}$ \\
\hline E & $\begin{array}{l}\text { Non peut être avec un fusain. c'est quoi un } \\
\text { fusain? }\end{array}$ & \\
\hline E & Bah c'est une sorte de petit truc noir. & \\
\hline EFS & Et on peut dessiner avec? & \\
\hline E & oui & \\
\hline
\end{tabular}

L'échange revient ensuite sur la mise en intrigue de ce qui est représenté.

Tableau 5. Description et mise en intrigue (suite)

\begin{tabular}{|l|l|l|}
\hline E & $\begin{array}{l}\text { Je peux montrer trois tableaux qui ont un truc en } \\
\text { commun. Ben on dirait que la mariée là elle est là, et que } \\
\text { elle, elle est là, et là. }\end{array}$ & $\begin{array}{l}\text { L'élève organise les mises en } \\
\text { récit précédentes, cherche une } \\
\text { cohérence narrative }\end{array}$ \\
\hline EFS & Alors pourquoi? & justification \\
\hline E & Elle a un voile sur la tête. & \\
\hline
\end{tabular}




\begin{tabular}{|l|l|l|}
\hline EFS & Là elle a un voile sur la tête. & \\
\hline E & $\begin{array}{l}\text { Et là aussi, mais elle a tout tout complètement le drap } \\
\text { sur elle comme si elle se mariait et on reconnait un peu } \\
\text { la couronne de fleurs sur la tête. Sur le côté. }\end{array}$ & $\begin{array}{l}\text { Justifie l'hypothèse } \\
\text { interprétative en convoquant } \\
\text { les référents }\end{array}$ \\
\hline EFS & $\begin{array}{l}\text { On reconnait un peu la couronne de fleurs sur le côté. } \\
\text { Eonc tu penses que c'est la même personne qui est ici. } \\
\text { Et alors toi tu penses que c'est une mariée. Une mariée ? }\end{array}$ & \\
\hline E & elle a des fleurs & \\
\hline
\end{tabular}

Lors de cette médiation, l'EFS médiatrice qui ne disposait d'aucune information précise sur le projet et l'intention de l'auteure a accueilli les propositions des élèves en les entrainant à développer, et étayer leur propos. Un récit s'est progressivement construit dans le petit groupe d'élèves en organisant la lecture dans l'ordre d'accrochage des gravures de gauche à droite. Mise en récit possible qui ne coïncide cependant pas exactement avec le projet de l'auteure qui est avant tout de réaliser une composition visuelle équilibrant les noirs et les blancs dans un univers fantomatique imprégné des contes. La confrontation de l'intention de l'auteur avec les hypothèses des élèves permettra d'enrichir la réception première et engagera le débat sur le fait qu'une œuvre peut recevoir plusieurs significations.

Les quelques fragments présentés ci-dessus donnent un aperçu de la façon dont la parole sur l'œuvre s'est construite dans le cadre prévu par l'enseignant. Dans le corpus recueilli lors de la visite de l'exposition «Phares » et lors des visites au Préau, la description et la narration organisées à partir de ce que l'élève voit représentent les genres discursifs dominants. L'appréciation artistique sur la façon de faire, les techniques employées, le temps de réalisation sont également récurrentes, l'expression d'une appréciation esthétique, d'un jugement de gout apparaissent mais restent au niveau du constat: l'argumentation est peu développée. La faiblesse du jugement de valeur argumenté tient en grande partie au manque de référents liés à la spécificité du domaine des arts. L'adhésion des membres du groupe à une idée exprimée est due à la reformulation et la répétition du point de vue de celui qui prend la parole. Des hypothèses interprétatives sont engagées mais elles n'aboutissent pas toujours.

\section{Perspectives}

Que nous disent les données recueillies? Les rencontres avec l'œuvre en musée ou galerie dans les dispositifs réalisés, comme nous l'avons dit, ne sont pas des rencontres entièrement libres et spontanées, mais les scenarios de médiation mis en place dans le cadre de la recherche ont visé à laisser le maximum d'autonomie dans les échanges. Les échanges et interactions entre élèves révèlent qu'il y a plusieurs niveaux de rencontre qu'il s'agisse de l'expression de l'émotion (en lien avec le vécu de l'élève ou ses connaissances), de tentatives d'interprétation (qui se traduisent par des mises en récit ou des descriptions) du discours d'expert quand il s'agit d'évoquer les techniques de l'artiste, ou du jugement esthétique. 
l'enseignant d'identifier les niveaux de rencontre et les formes langagières qu'elles prennent. Ces observations permettent ensuite de définir des objets d'étude en classe. Dire l'émotion, c'est identifier l'effet que produit l'œuvre et disposer du bagage lexical pour dire l'émotion. Tenir un discours d'expert c'est maitriser le langage technique de l'histoire des arts et des techniques artistiques. Partager au sein d'un groupe un jugement de gout c'est pouvoir le formuler, l'argumenter, le partager.

Accompagner les élèves dans ces directions par des situations didactisées en classe c'est leur permettre de passer progressivement d'une appréhension première de l'œuvre essentiellement sensorielle et émotive à une appréhension développée, augmentée, c'està-dire qui concilie à la fois l'expression des émotions et l'investissement intellectuel : une émotion réfléchie.

\section{BIBLIOGRAPHIE}

AUJALEU, E. (1997). « La sensibilité esthétique et le jugement de goût ». Tréma 11, p. 33-52. En ligne : http://trema.revues.org/1921.

BARYGA, P. (2015). « Comprendre une œuvre d'art. L'expertise des élèves et ses présupposés esthétiques ». Spirales 56, p. 97-106. En ligne : http://www.persee.fr/doc/ spira_0994-3722_2015_num_56_1_1010.

BORDEAUX, M.-C. (2008). « La médiation culturelle en France, conditions d'émergence, enjeux Politiques et théoriques ». Actes du Colloque international sur la médiation culturelle, Montréal, 4-5 décembre 2008. En ligne : https://www.lmac-mp.fr/telecharger.php?id_doc=192.

BUCHETON, D. \& SOULÉ Y. (2009). « Les gestes professionnels et le jeu des postures de l'enseignant dans la classe : un multi-agenda de préoccupations enchâssées ». Éducation et didactique 3, 3, p. 29-48. En ligne : http://journals.openedition.org/educationdidactique/543.

CoAvoux, S. (2012). « Compétence artistique, réception et démocratisation ». Marges 15, p. 69-80. En ligne : http://journals.openedition.org/marges/355.

DERONNE, C. (2011). « Former des enseignants à exprimer leur expérience singulière d'une œuvre littéraire ou plastique ». Repères 43, p. 103-126. En ligne : http://journals.openedition.org/ reperes/222.

DERONNE, C. (2012). «Quels effets des gestes de l'enseignant sur la rencontre avec l'œuvre?» In : Chabanne, J.-C., Parayre, M. \& Villagordo, É. (dirs), La rencontre avec l'oeuvre. Éprouver, pratiquer, enseigner les arts et la culture. Paris : L'Harmattan, p. 117-132.

DUfAYS, J.-L., GEMENNE, L. \& LEDUR, D. (2005) [1996]. Pour une lecture littéraire. Bruxelles : De Boeck. 
DUMORTIER, J.-L. (2006). « Conduite esthétique, jugement esthétique et écriture de soi ». Repères 34, p. $185-214$.

EVERAERT-DESMEDT, N. (2010). « Les voies de l'interprétation : un rendez-vous à l'aveugle ». In : Gaudez, Fl. (dir.), La Culture du texte. Approches socio-anthropologiques de la construction fictionnelle. Paris : L'Harmattan, p. 53-71.

GOODMAN, N. (2006) [1978]. Manières de faire des mondes. Trad. de l'américain par M.-D. Popelard. Paris : Gallimard

MARTIN, J.-H. (2012). « Théâtre du monde ». In : Martin, J.-H., L'art au large. Paris : Flammarion.

RANCIÈRE, J. (2008). Le spectateur émancipé. Paris : Éd. La Fabrique.

SCHAEFFER, J.-M. (2015). L'Expérience esthétique. Paris : Gallimard.

TALON-HUGON, C. (2004). « L'émotion poétique ». Noesis 7. En ligne : http://

journals.openedition.org/noesis/30.

TALON-HUGON, C. (2016). « De la pathétique artistique à l'émotion esthétique ». L'Atelier du Centre de recherches historiques 16. En ligne : http://journals.openedition.org/acrh/7301.

\section{NOTES}

1. Quatre enseignants du premier degré auxquels se sont ajoutés 4 étudiants fonctionnaires stagiaires master MEEF (métiers de l'enseignement, de l'éducation et de la formation) en 2015-1206.

2. «Phares », du 14 février 2014 au 15 février 2016, Centre Pompidou-Metz. En ligne : http:// www.centrepompidou-metz.fr/phares.

3. Code RNCP : 14595. Accès : http://www.rncp.cncp.gouv.fr./

\section{RÉSUMÉS}

Cet article propose d'explorer les significations de ce que recouvre «la rencontre avec les œuvres d'art » que préconisent les programmes de l'enseignement primaire (2015). Dans le cadre d'une recherche en lien avec l'Institut français d'éducation, notre équipe a conçu plusieurs situations didactisées de l'expérience de la rencontre dans les lieux dédiés à la diffusion de la création (musée et galerie). Nous analysons dans les pages qui suivent ce que nous disent les discours des élèves de leur relation à l'œuvre d'art à travers trois dispositifs spécifiques centrés sur les interactions élèves/élèves et médiateur/élèves. La pluralité des pratiques langagières observées (exprimer une émotion, décrire ce qu'on voit, le mettre en intrigue, apprécier une forme, une couleur, interpréter, commenter, mettre en réseau, contextualiser, etc.) traduit la pluralité des rencontres possibles et interroge les finalités des types de dispositifs didactiques de la rencontre.

This article proposes to explore the meanings of the "encountering a work of art" that are advocated by the curricula of primary education (2015). As part of a research project linked to the French Institute of Education, our team has designed several didactical situations of the 
experience of encountering a work of art in places dedicated to the diffusion of creation (museum and gallery). In the following pages, we analyze what students' speeches tell us about their relationship to the work of art through three specific mechanisms centered on pupil-pupil and pupil-mediator interactions. The plurality of the observed linguistic practices (expressing an emotion, describing what is seen, putting it into intrigue, appreciating a form, a color, interpreting, commenting, networking, contextualizing, etc.) translates the plurality of possible encounters and questions the aims of the types of didactic devices of the encounter.

INDEX

Mots-clés : rencontrer une œuvre d'art, parler d'une œuvre d'art, émotion, réception, plaisir, jugement, médiation

Keywords : encountering a work of art, talking about a work of art, emotion, reception, pleasure, judgment, mediation

\section{AUTEUR}

\section{CHRISTINE DERONNE}

Université de Lorraine, ESPÉ, CREM, EA 34 76, F-54000, France 